IRSTI 29.19 .22

\title{
Silicon nanocrystals: physical properties and potential biomedical applications
}

\author{
V. Yu. Timoshenko ${ }^{1,2,3 *}$ \\ ${ }^{1}$ Lomonosov Moscow State University, Physics Department, Leninskie Gory 1, 119991, Moscow, Russia, \\ ${ }^{2}$ National Research Nuclear University "MEPhI”, Nanotheranostics Laboratory, \\ 31 Kashirskoe Sh., 115409 Moscow, Russia, \\ ${ }^{3}$ Lebedev Physical Institute of the Russian Academy of Sciences, \\ 53 Leninskiy Prosp., 119991, Moscow, Russia \\ *e-mail: timoshen@physics.msu.ru
}

\begin{abstract}
Silicon ( $\mathrm{Si}$ ) nanocrystals (NCs) possess photoluminescence at room temperature and promising properties for biomedical applications as bioimaging and various therapeutic modalities. The photodynamic therapy application is based on an ability of Si-NCs to sensitize the generation of reactive oxygen species under illumination. The sonodynamic therapy with Si-NCs can be realized due to their properties to activate the cavitation followed by destruction of cancer cells and tumors. Furthermore, Si-NCs in aqueous media under electromagnetic radiofrequency irradiation can activate the heat release that is promising for the hyperthermia treatment of cancer. This paper reviews the physical properties of Si NCs, which are essential for their applications both in medical diagnostics and therapy. In the paper the properties of SiNCs and nanoparticles (NPs) are analyzed to reveal their potential for biomedical applications. Besides the data on physical properties of Si-NCs and NPs the results of biomedical investigations of these nanomaterials are also discussed.
\end{abstract}

Keywords: silicon nanocrystals, photoluminescence, bioimaging, biomedical applications.

PACS numbers: $61.46 .+\mathrm{w}, 78.55 .-\mathrm{m}$.

\section{Introduction}

Silicon ( $\mathrm{Si}$ ) is one of the most abundant elements in Earth's crust (27.7\% by weight) and its crystalline form c-Si is the main functional material for modern micro- and optoelectronics. Recently, $\mathrm{Si}$ nanocrystals (NCs) have attracted much attention due to their physical properties and potential applications in optoelectronics, sensorics and biomedicine [1-5]. The rapid development in Si-NC applications is favorable because of their quite low toxicity and an ability to be eliminated from the body by dissolving into orthosilicic acid [6,7]. SiNCs are proposed to be used in cancer therapy as photosensitizers [3] and sensitizers of ultrasound radiation [8, 9], high frequency electromagnetic field [10], and drug carriers [11-13]. It was shown that Si-NPs are promising for both cancer therapy and diagnostics, i.e. theranostics [14-17]

Many years ago, porous $\mathrm{Si}$ (por-Si) was invented and investigated as a new morphological form of silicon formed by electrochemical etching of bulk crystalline silicon (c-Si) wafers in hydrofluoric acid solutions [18]. Now it is known that por-Si formed from substrates of lightly doped p-type c-Si wafer is microporous, i.e. the mean pore size does not exceed $2 \mathrm{~nm}$ [19]. For high pore density, which can be achieved at certain etching regime, microporous por-Si consists of interconnected $\mathrm{Si}-\mathrm{NCs}$ with average sizes varied from 1 to $5 \mathrm{~nm}$, depending on the preparation conditions [20]. The quantum confinement for charge carriers in such small $\mathrm{NC}$ results in an increase of the energy gap [21-23]. The confinement effect is found to be strongest for spherical clusters of $\mathrm{Si}$ atoms, i.e. for smallest $\mathrm{NCs}$ with size restrictions in three dimensions [24]. Recently, chemically pure spherical Si-NCs were formed by pulsed lased ablation in inert gas or in water and various biomedical applications of the prepared NCs were proposed $[16,17]$.

In the present paper the properties of $\mathrm{Si}-\mathrm{NCs}$ and nanoparticles (NPs) are analyzed to reveal their potential for biomedical applications. Besides the 
data on physical properties of Si-NCs and NPs the results of biomedical investigations of these nanomaterials are also discussed.

\section{Optical absorption and photoluminescence}

Due to the quantum confinement and size distribution of Si-NCs, the effective band-gap energy of por-Si ranges typically from $1.2 \mathrm{eV}$ up to $2.5 \mathrm{eV}$ as it was theoretically shown in Ref. [24]. Experimental data of the absorption coefficient of $\mathrm{Si}-\mathrm{NCs}$ in mesoporous $\mathrm{Si}$ demonstrate that for the photon energy above $3 \mathrm{eV}$ the main contribution to the absorption is due to the transitions above the direct gap of c-Si and then the spectrum is similar to that of $\mathrm{c}-\mathrm{Si}[1,2]$. In this spectral range the absorption coefficient can be well described by the effective medium approximation [25]. The shift of the absorption curve to higher energies is due to reduced density and quantum confinement in SiNCs. Only at high energies when all NCs are absorbing in the range of a high density of states this discrepancy becomes smaller and, finally, vanishes at the energy of the direct transitions [21]. Note the absorption coefficient of por-Si shows the continuous spectral dependence (see Figure 1), which is important for the photosensitizing properties under excitation in the broad spectral range.

While the photoluminescence (PL) of por-Si is sensitive to details of the electrochemical formation and storage there are unambiguous evidences of the quantum confinement effect in Si-NCs on the PL band position $[1,2]$. For example, it is possible to tune the PL band position of por-Si in the spectral range from 1.1 to $2.5 \mathrm{eV}$ by using different $\mathrm{c}-\mathrm{Si}$ substrates and etching conditions (Fig.2). The PL properties of por-Si are also dependent on defects and contaminations, which can take place on highly developed surface of Si-NCs [1,2]. The influence of defect states on the optical and electronic properties of Si-NCs was confirmed by many experimental methods as optical absorption spectroscopy $[21,24]$, PL and photoacoustics spectroscopy [26-28] as well as electron paramagnetic resonance (EPR) measurements [29-31].

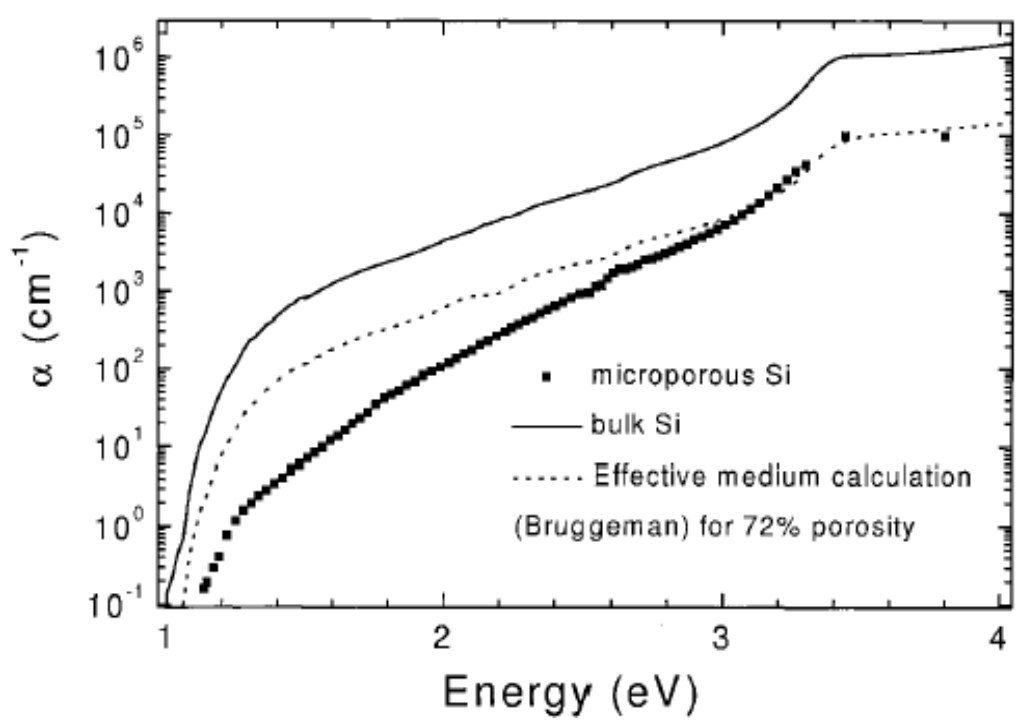

Figure 1 - Spectra of the absorption coefficient of microporous Si (dots) and bulk c-Si (solid line) at $\mathrm{T}=300 \mathrm{~K}$. Dashed line: effective medium calculations using the Bruggeman approach for $72 \%$ porosity (Ref. [21]) 


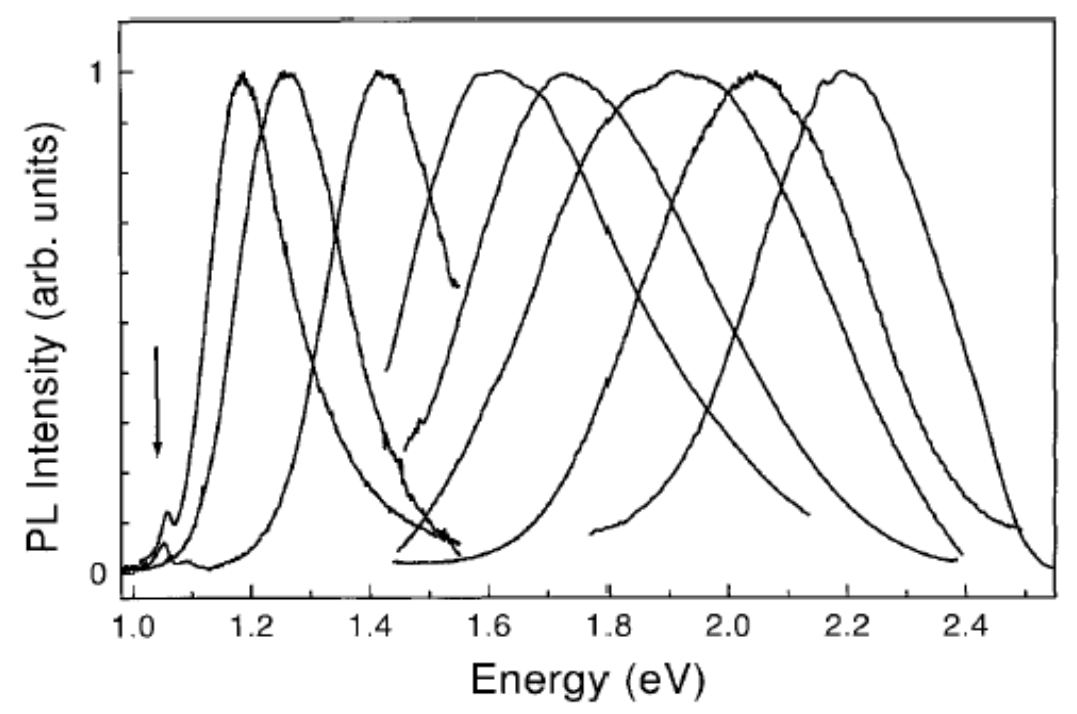

Figure 2 - PL spectra of por-Si prepared with various etching parameters.

The arrow indicates the spectral position of the lowest energy free exciton transition in c-Si (Ref. [21])

It is established that the Coulomb interaction between photoexcited electrons and holes in a small Si-NC influences strongly their energies and then the PL spectrum $[23,24]$. The attractive Coulomb interaction for charge carriers in the Si-NC results in a formation of excitons with relatively large binding energies of about hundreds of meV, which determine possibilities for the excitons to be stable at room temperatures [22]. Furthermore, the exchange interaction in an exciton can remove the spin degeneracy for the exciton states. It results in two possible spin configurations for the exciton in Si-NCs, which consist of optically active spinsinglet states and optically inactive spin-triplet ones. The triplet exciton state is lowered in energy in respect to the singlet state because of the electronhole exchange interaction [23]. Despite a small singlet-triplet splitting being in the range from 1 to $30 \mathrm{meV}$ the main part of excitons $(75 \%)$ reside in the threefold degenerated triplet state even at elevated temperatures [21].

\section{Photosensitizing properties}

Singlet oxygen $\left({ }^{1} \mathrm{O}_{2}\right)$ is known to be an excited form of molecular oxygen $\left(\mathrm{O}_{2}\right)$, which mediates important biochemical processes in living organisms [32]. The biochemical reactivity of ${ }^{1} \mathrm{O}_{2}$ is used in socalled photodynamic therapy (PDT) of cancer, which is based on a combination of photoactive drug and light treatment of tumors [33]. According to the quantum mechanics the relaxation of singlet states back to the ground (triplet) state is "spin forbidden" and thus ${ }^{1} \mathrm{O}_{2}$ is characterized by rather long lifetime [35], which is favorable for transferring the excess energy to another molecule. The most useful in biomedical applications and widely used procedure of the ${ }^{1} \mathrm{O}_{2}$ generation is a photosensitization, which includes a certain substance called "photosensitizer", e.g. dye molecules $[34,35]$. It was found that Si-NCs in asprepared por-Si were able to photosensitize the ${ }^{1} \mathrm{O}_{2}$ generation [36]. The observed efficient photosensitization was explained by the energy transfer from excitons in Si-NCs to $\mathrm{O}_{2}$ molecules adsorbed on the surface of Si-NCs [37]. This process is favorable because of highly developed surface area of the order of $10^{2} \div 10^{3} \mathrm{~m}^{2} / \mathrm{g}$ of small Si-NCs $[19,20]$ and long radiative lifetimes $\left(10^{-}\right.$ ${ }^{5} \div 10^{-3} \mathrm{sec}$ ) of excitons confined in the Si-NCs [21].

A quenching of the exciton photoluminescence (PL) of Si-NCs in por-Si with adsorbed $\mathrm{O}_{2}$ molecules was used to clarify the energy transfer mechanism [37,38]. While the PL quenching is only an indirect evidence of the ${ }^{1} \mathrm{O}_{2}$ formation, the direct and quantitative data can be obtained by analyzing the radiative decay of ${ }^{1} \mathrm{O}_{2}$ molecules at $0.98 \mathrm{eV}$ $(1270 \mathrm{~nm})$ [37,39]. In fact, the ${ }^{1} \mathrm{O}_{2}$ photosensitization by $\mathrm{Si}-\mathrm{NC}$ was quantified by means of the luminescence spectroscopy at $0.98 \mathrm{eV}$ 
[37]. On the one hand, a lot of the experiments on the ${ }^{1} \mathrm{O}_{2}$ luminescence demonstrates the efficient generation of ${ }^{1} \mathrm{O}_{2}$ by the energy transfer from SiNCs [36]. On the other hand, a comparison of the ${ }^{1} \mathrm{O}_{2}$ luminescence intensity for Si-NCs and for the standard photosensitizers as porphyrins gave a strong evidence of a deactivation (quenching) of ${ }^{1} \mathrm{O}_{2}$ molecules in Si-NC structures [40], which limits significantly the net concentration of ${ }^{1} \mathrm{O}_{2}$ sensitized by Si-NCs. The EPR technique can provide information about the ${ }^{1} \mathrm{O}_{2}$ concentration in different reactions [34, 35]. The X-band EPR spectroscopy of photoexcited $\mathrm{Si}-\mathrm{NCs}$ in $\mathrm{O}_{2}$ atmosphere gave estimates of the concentration of photosensitized ${ }^{1} \mathrm{O}_{2}$ molecules to be about $10^{18} \div 10^{19} \mathrm{~cm}^{-3}$ under excitation with photon flux of about $10^{19} \div 10^{20} \mathrm{~cm}^{-2}$ $[30,31]$. The ${ }^{1} \mathrm{O}_{2}$ photosensitization was observed for por-Si NPs dispersed in different oxygen-saturated organic and inorganic liquids [38] as well in water [39]. Furthermore, the biomedical experiments in vitro demonstrated an effect of the strong suppression of the cancer cell proliferation by introducing photoexcited por-Si NPs [3].

The first evidence of the photosensitized property of Si-NCs was observed at cryogenic temperatures $[5,6]$. The investigated samples of por-Si were prepared by standard electrochemical etching of (100)-oriented boron-doped c-Si wafers with a resistivity of $10-20 \Omega^{*} \mathrm{~cm}$ in a solution of hydrofluoric acid. he prepared free standing por-Si layers were dried in air for several hours. Then, the prepared layers were milled to get powder assembled from por-Si NPs and their agglomerates. The powder was kept in a vacuum chamber in oilfree vacuum with a residual pressure of about $10^{-5}$ Torr. The exciton PL spectrum of por-Si measured in vacuum at low temperature is characterized by a broad, featureless emission band located in the visible region (dashed line in Figure 3). The spectral position and bandwidth reflect the wide band-gap distribution of the Si-NC assembly. Intrinsic defects as Si dangling bonds [31] seem to be responsible for the infrared PL band below $1 \mathrm{eV}$ [21]. The adsorption of $\mathrm{O}_{2}$ on the surface of Si-NCs resulted in a strong suppression of both the exciton PL and the defect PL band (solid line in Figure 3). The PL quenching by $\mathrm{O}_{2}$ adsorption is maximal for the PL photon energies above $1.63 \mathrm{eV}$. The latter value corresponds to the ${ }^{3} \Sigma \rightarrow{ }^{1} \Sigma$ transition energy in $\mathrm{O}_{2}$ molecule. At the same time the PL line at $0.98 \mathrm{eV}$, which is related to the radiative transition ${ }^{1} \Delta \rightarrow{ }^{1} \Sigma$, appeared after oxygen adsorption on $\mathrm{Si}-\mathrm{NCs}$ (see inset in Figure 3). These spectroscopic data give direct evidences of the ${ }^{1} \mathrm{O}_{2}$ photosensitization by SiNCs. Detailed description of the energy-transfer mechanism responsible for the photosensitized generation of ${ }^{1} \mathrm{O}_{2}$ by Si-NCs can be obtained from the spectral dependence of the PL quenching strength, which can be defined as follows [36]:

$$
Q=I_{P L^{v a c}} / I_{P L^{o x}},
$$

where $I_{\mathrm{PL}}{ }^{\text {vac }}$ and $I_{\mathrm{PL}}{ }^{\text {ox }}$ are the PL intensities of Si$\mathrm{NCs}$ in vacuum and in oxygen ambient, respectively. The efficiency of the energy transfer from excitons in Si-NCs to $\mathrm{O}_{2}$ molecules can be obviously calculated by using the following expression [28]:

$$
\eta=1-Q^{-1} .
$$

The spectra of $Q\left(h v_{P L}\right)$ and $\eta\left(h v_{P L}\right)$ demonstrate both the main maximum at $1.63 \mathrm{eV}(Q>>1, \eta \sim 1)$ and an additional maximum at $h v_{P L}$ of about 57 $\mathrm{meV}$ below the ${ }^{1} \Delta$ state energy as well as numerous maxima separated by energy of about $63 \mathrm{meV}$ [36, 37]. These maxima correspond to the energy transfer from Si-NCs with band gaps, which do not match resonantly the excitation energies of the singlet states of $\mathrm{O}_{2}$. A local maximum separated by $57 \mathrm{meV}$ from the main maximum at $1.63 \mathrm{eV}$ is an evidence of the indirect radiative transition in $\mathrm{Si}$ NCs where the participation of the momentum conserving TO phonon with energy of $57 \mathrm{meV}$ is required [21]. The excess of the exciton energy with respect to the energies of the ${ }^{1} \Delta$ and ${ }^{1} \Sigma$ states is released the emission of TO phonons with energy of $63 \mathrm{meV}$ [36]. 


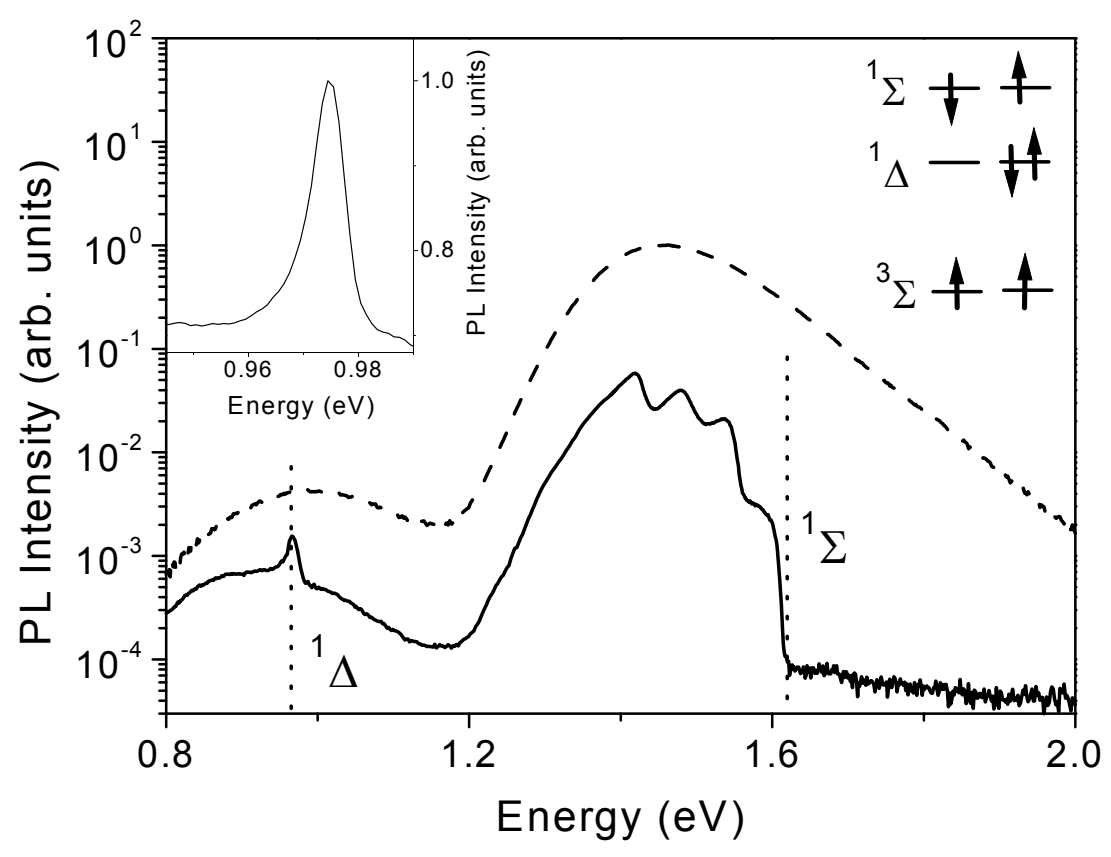

Figure 3 - PL spectra of por-Si in vacuum (dashed line) and in the presence of adsorbed oxygen molecules (solid line). Transition energies from the excited states $\left({ }^{1} \Delta,{ }^{1} \Sigma\right.$ ) of ${ }^{1} \mathrm{O}_{2}$ to the ground state are indicated by dashed vertical lines. Spin configurations and spectroscopic labeling of molecular oxygen states are shown. Inset depicts

the PL line related to the ${ }^{1} \Delta \rightarrow{ }^{1} \Sigma$ transition in $\mathrm{O}_{2}$. Temperature $\mathrm{T}=5 \mathrm{~K}$, excitation at $2.41 \mathrm{eV}$ (Ref. [37])

The dynamics of the energy transfer can be deduced from the PL transients of Si-NC in the presence of adsorbed $\mathrm{O}_{2}$ molecules. The energy transfer time from one $\mathrm{Si}$ nanocrystal to a single $\mathrm{O}_{2}$ molecule is not accessible experimentally, since a large number of Si-NCs contributes to the PL at certain emission energy. At low temperatures in vacuum the $\mathrm{PL}$ decay times, $\tau_{\mathrm{PL}}$, is approximately equal to the radiative lifetime of excitons in Si-NCs, $\tau_{\mathrm{ex}}$, and it is about $\sim 10^{-3} \mathrm{~s}$ independently on the PL photon energy [21]. The PL quenching by $\mathrm{O}_{2}$ adsorption is accompanied by a significant decrease of the PL decay time because the adsorbed $\mathrm{O}_{2}$ molecules on the Si-NC surface represent a channel for the nonradiative recombination of the excitons. The spectral dependence of $\tau_{\mathrm{PL}}$ follows the quenching strength spectrum. The shortest $\tau_{\mathrm{PL}}$ is observed for the Si-NCs, which transfer their excitation most efficiently $[36,37]$. Increasing the concentration of adsorbed $\mathrm{O}_{2}$ molecules results in a stronger PL quenching and further decrease of $\tau_{\mathrm{PL}}$ [37]. The time resolved PL studies performed with nanosecond time resolution confirmed this spectral behavior of the exciton lifetimes of $\mathrm{Si}-\mathrm{NCs}$ in oxygen ambient (see Figure 4).

Besides the quenched exciton PL in por-Si with adsorbed $\mathrm{O}_{2}$ molecules there are infrared PL bands related to defects in Si-NCs and radiative transitions in the photosensitized ${ }^{1} \mathrm{O}_{2}$ (see Figure 3). The relevant time scale of the infrared PL was determined from time-resolved measurements. Inset of Figure 4 shows the transients of the defect-related emission for por-Si in vacuum detected at the photon energy of 0.953 and $0.973 \mathrm{eV}$. The rise time coincides within the response time of the used experimental setup ( $\sim 100 \mathrm{~ns})$ and yields an upper limit for the time of carriers capture to defect states [21]. The nonexponential decay proceeds on a microsecond time scale and does not vary with PL photon energy. In the presence of adsorbed $\mathrm{O}_{2}$ molecules the radiative decay of the ${ }^{1} \Delta$ state of ${ }^{1} \mathrm{O}_{2}$ is observed at detection energy of $0.953 \mathrm{eV}$. The initial fast decay is attributed to the defect-related PL background. The slow component with the lifetime $\tau_{\Delta} \approx 0.5 \mathrm{~ms}$ accounts for the ${ }^{1} \Delta \rightarrow{ }^{3} \Sigma$ transition in $\mathrm{O}_{2}$. This lifetime is significantly shorter 
that the radiative lifetime of a free $\mathrm{O}_{2}$ molecule due to the interaction with Si-NCs. The same interaction should also influence the radiative lifetime of adsorbed ${ }^{1} \mathrm{O}_{2}$ molecules. Firstly, the quantum yield $\eta_{r} \approx 7 \cdot 10^{-5}$ of the radiative decay of the ${ }^{1} \Delta$ state is estimated from the comparison from the integral intensity of the exciton PL band. Then $\tau_{r} \approx 7 \mathrm{~s}$ is obtained by using the simple relation $\tau_{r}=\tau_{\Delta} / \eta_{r}$. On the one hand, the obtained $\tau_{r}$ is much shorter than the undisturbed radiative lifetime of about $50 \mathrm{~min}$ [34]. On the other hand, $\tau_{r} \approx 7 \mathrm{~s}$ is close to the radiative lifetimes of ${ }^{1} \mathrm{O}_{2}$ in different solvents [35]. Therefore, Si-NCs act as both the photosensitizer and a quencher of ${ }^{1} \mathrm{O}_{2}$.

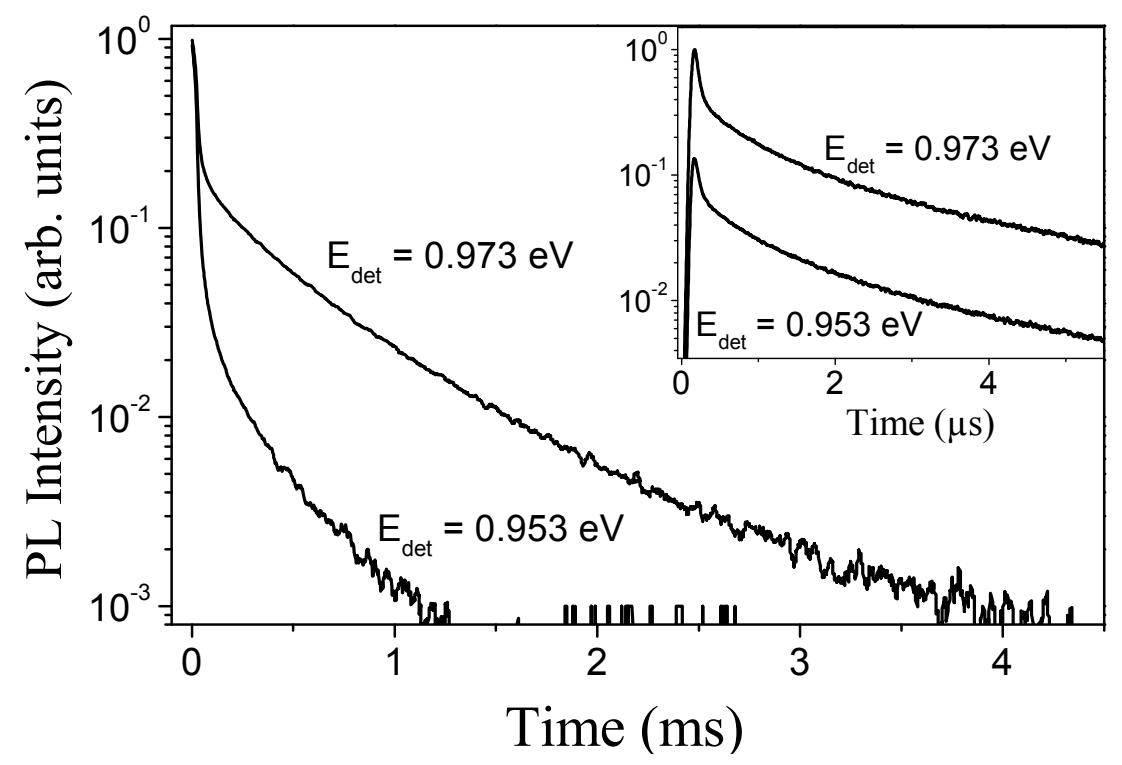

Figure 4 - Transients of the infrared $\mathrm{PL}$ of ${ }^{1} \mathrm{O}_{2}$ (detection energy $\mathrm{E}_{\mathrm{det}}=0.973 \mathrm{eV}$ ) and defect-related $\mathrm{PL}$ at $\mathrm{h} \nu_{\mathrm{PL}}=0.953 \mathrm{eV}$ of por-Si with adsorbed $\mathrm{O}_{2}$ molecules at $\mathrm{T}=5 \mathrm{~K}$. Inset: PL transients of the defect-related PL of por-Si in vacuum (Ref. [37])

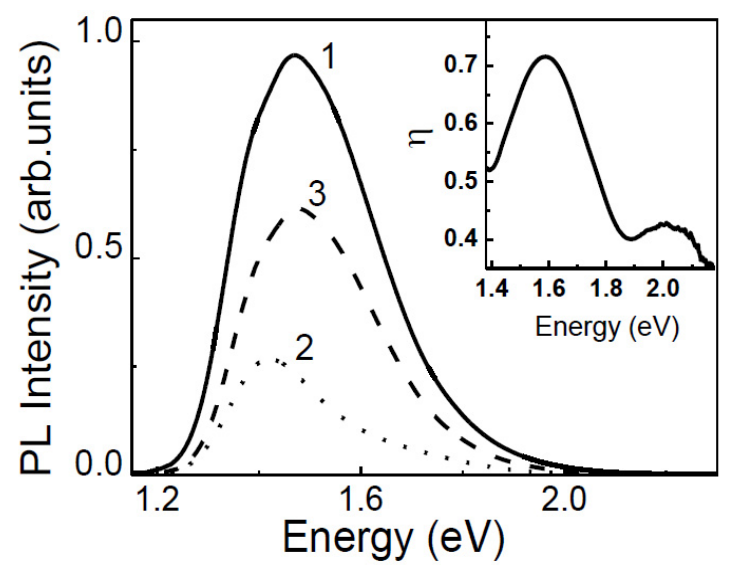

Figure 5. Spectra of the room temperature PL of por-Si in vacuum (1), in oxygen ambient at $p=760$ Torr (2) and just after evacuation (3). Inset shows the spectrum of the energy transfer (Ref. [28]) 
While the above discussed PL experiments at cryogenic temperatures are important to clarify details of the photosensitization of ${ }^{1} \mathrm{O}_{2}$ by Si-NCs, the ${ }^{1} \mathrm{O}_{2}$ generation at room temperature is of most practical interest. Contrary to cryogenic temperatures, the optimal interaction conditions for $\mathrm{Si}-\mathrm{NC} / \mathrm{O}_{2}$ system are not fulfilled at elevated temperatures. Because of the thermally activated desorption a small spatial separation is realized only during a short time of collisions between $\mathrm{O}_{2}$ molecules and Si-NC's surface. Additionally, the exciton lifetime and the occupation number of the spin-triplet state of the exciton decrease with rising temperature [21]. It results in a weaker PL quenching, which scales with the collision rate, i.e., the gas pressure [37], and in a broad spectral resonance of the energy transfer to the ${ }^{1} \sum$ state as it is shown in Figure 5. The corresponding spectrum of $\eta$ is presented in the inset of Fig.5. On the one hand, the maximal value of $\eta \approx 0.7$, which occurs at $1.63 \mathrm{eV}$, indicates the rather efficient energy transfer from excitons in $\mathrm{Si}-\mathrm{NCs}$ with resonant energies to $\mathrm{O}_{2}$ molecules. On the other hand, the spectrally averaged efficiency of the energy transfer for por-Si, which can be obtained by simple integration of $\eta\left(h v_{P L}\right)$, does not exceed 0.5. Taking into account that the quantum efficiency of the exciton PL is typically smaller $10 \%$, one can estimate the quantum efficiency of the ${ }^{1} \mathrm{O}_{2}$ generation at room temperature to be lower $5 \%$. Note, the dependence of the ${ }^{1} \mathrm{O}_{2}$ generation efficiency on the $\mathrm{O}_{2}$ ambient pressure at room temperature is well described by the Langmuir approach for molecule adsorption [28]. The good agreement between the experimental data and Langmuir model indicates that the amount of the photosensitized singlet oxygen is almost proportional of the number of adsorbed oxygen molecules. This fact has to be taken into account for different applications of the photosensitizing properties of Si-NCs.

Besides the characteristic energy of about 1.63 $\mathrm{eV}$ (the ${ }^{1} \Sigma \rightarrow{ }^{3} \Sigma$ transition energy in $\mathrm{O}_{2}$ molecule), the spectrally selective quenching of PL intensity of por-Si can demonstrate an additional maximum at photon energy of about 2-2.2 eV (see inset in Fig.5). The high energy quenching maximum is usually observed for por-Si samples with a broad distribution of Si-NCs sizes and surface conditions, e.g. for por-Si powders obtained by mechanical grinding of por-Si films or by stain etching of polycrystalline Si powder [39]. The origin of the high energy quenching can be related to the formation of superoxide radicals, i.e. $\mathrm{O}_{2}^{-}$[35]. There are no unambiguous evidences of this characteristic energy of the $\mathrm{O}_{2}^{-}$formation. But the latter process is known to be more energy consumed than the ${ }^{1} \mathrm{O}_{2}$ generation. The PL quenching at 2-2.2 eV can be also explained by the charging of $\mathrm{Si}-\mathrm{NCs}$ due to the $\mathrm{O}_{2}^{-}$formation on the Si-NC's surface. The positively charged volume of Si-NCs results in an increase of the nonradiative Auger recombination [28].

Additional information about the ${ }^{1} \mathrm{O}_{2}$ photosensitization at room temperature is obtained from the PL transient measurements. Figure 6 presents several PL transients of por-Si in gaseous oxygen ambient [37]. The PL transients are not actually mono exponential (see Figure 6). However, for longer times (typically, $>50 \mu \mathrm{s}$ after the excitation laser pulse) they can be approximated by the exponential function as follows:

$$
I_{P L}(t)=I_{P L}(0) \exp \left(-t / \tau_{P L}\right)
$$

where $\tau_{P L}$ is the mean PL lifetime, and $I_{P L}(0)$ is the PL intensity just after excitation. The values of $\tau_{P L}$ for the samples in vacuum are found to be about tens microseconds that is in good agreement with the singlet lifetimes of excitons in Si-NCs [21].

The admission of $\mathrm{O}_{2}$ causes a decrease of $\tau_{\mathrm{PL}}$ and this effect is stronger at higher oxygen pressure (see Figure 6). The strongest lifetime shortening occurs at the PL energy of $1.6 \mathrm{eV}$, which correlates with the spectral maximum of the PL intensity quenching. The PL lifetime shortening after oxygen adsorption was nearly reversible at low oxygen pressure [28]. The detailed analysis of the PL transients indicates that the singlet oxygen-induced defect formation results mainly in a decrease of the number of luminescent Si-NCs. It can be understood by taking into account the short lifetime $(<1 \mu \mathrm{sec})$ of the nonradiative recombination of photoexcited charge carriers in Si-NCs with dangling bonds [36]. 


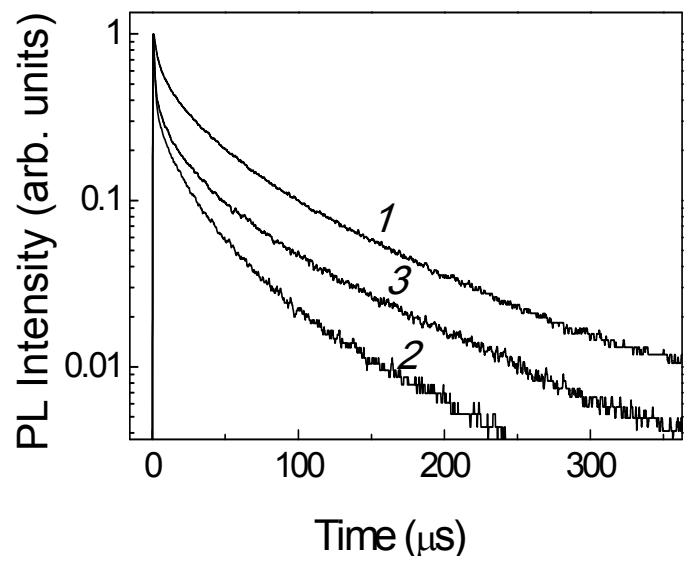

Figure 6 - Transients of the room temperature PL at $1.63 \mathrm{eV}$ of por-Si in vacuum (1), in oxygen ambient at $p=760$ Torr (2) and just after evacuation (3)

Due to the fast nonradiative relaxation of ${ }^{1} \mathrm{O}_{2}$ in water [35] it is practically impossible to detect the ${ }^{1} \mathrm{O}_{2}$ luminescence in aqueous solutions. In this case the ${ }^{1} \mathrm{O}_{2}$ photosensitization can be indirectly probed by monitoring the intensity and lifetime of the exciton PL of Si-NCs. Figure 7 shows typical PL spectra of por-Si powder dispersed in oxygensaturated water and in water after $\mathrm{O}_{2}$ degassing. One can see that $\mathrm{O}_{2}$ dissolved in water caused the spectrally selective PL quenching (inset in Figure
7). Although the PL quenching strength in water is weaker, its spectral shape is similar to that measured in gaseous $\mathrm{O}_{2}$ ambient. Note that the relative contribution of the high-energy quenching at 2$2.2 \mathrm{eV}$ is stronger for por-Si in water than for the case of gaseous ambient. It implies that the Si-NCs charging or/and $\mathrm{O}_{2}^{-}$formation is more efficient in presence of polar molecules of water, which can decrease barriers for the charge trapping on the $\mathrm{Si}$ NC's surface.

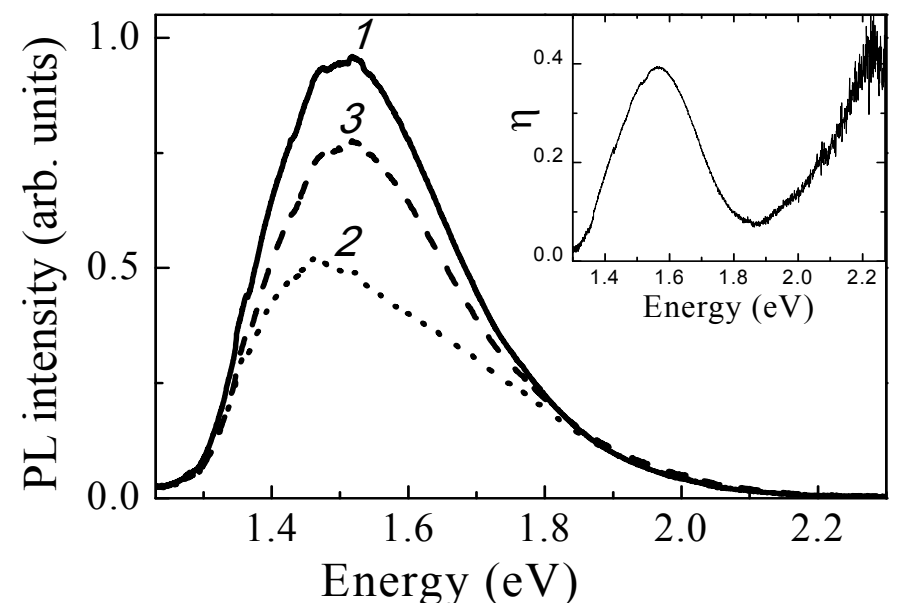

Figure 7 - Spectra of the room temperature PL of por-Si in water without (1) and with dissolved oxygen at $p=760$ Torr (2) and just after $\mathrm{O}_{2}$ degassing (3).

Inset shows the spectral dependence of the efficiency of energy transfer

The lifetime of the exciton PL of por-Si NPs dispersed in water was found to decrease after saturation of the solution by $\mathrm{O}_{2}$. The energy transfer times can be determined from transients of the exciton PL [28]. The spectral dependence of the energy transfer time was found to be similar to that in gaseous oxygen ambient. A slightly longer energy transfer time correlates with weaker quenching of 
the PL intensity of por-Si in water than that in gaseous $\mathrm{O}_{2}$. This fact indicates an influence of water molecules on the exciton recombination and/or energy transfer from the excitons to $\mathrm{O}_{2}$ molecules. Indeed, the energy transfer is mostly efficient for Si$\mathrm{NCs}$ with hydrogenated (H-terminated) surfaces, which are hydrophobic [19]. Therefore, Si-NCs dispersed in water should be partially oxidized that results in decreasing the ${ }^{1} \mathrm{O}_{2}$ photosensitization efficiency [37].

The oxidation of $\mathrm{Si}-\mathrm{NCs}$ in air or in aqueous solutions seems to be the main limiting factor to achieve the stable and efficient photosensitization of $\mathrm{O}_{2}$ generation [39]. The oxidation process can be also enhanced by ${ }^{1} \mathrm{O}_{2}$ photosensitized by por-Si [38]. In order to overcome this serious limitation for the application of Si-NCs as efficient photosensitizer of ${ }^{1} \mathrm{O}_{2}$ it is probably required a special procedure of controllable oxidation and/or surface modification of Si-NCs. It can be done, for example, by using rapid thermal annealing or laser irradiation.

The above discussed efficient photosensitization of ${ }^{1} \mathrm{O}_{2}$ by Si-NCs in different ambiences stimulates experiments on biomedical verification for the expected photodynamic action. It was found that photoexcited por-Si NPs, which consisted of aggregated $\mathrm{Si}-\mathrm{NCs}$, could suppress the proliferation (permanent division) of cancer cells [3]. For these biomedical experiments Si-NCs were prepared by using the conventional method of the electrochemical etching of $\mathrm{c}-\mathrm{Si}$ wafers in hydrofluoric acid solutions [19]. The obtained freestanding por-Si films were dried in air and then milled to get a powder of por-Si NPs. According to the Raman scattering data, the mean sizes of Si-NCs in the initial por-Si layers and in the powder formed from them were about of 3-4 $\mathrm{nm}$. Then the por-Si powder was dispersed in pure water bubbled with oxygen to get a homogeneous aqueous suspension. It was found that the exciton PL intensity of Si-NCs in oxygen-saturated water decreases by 1.5-2 times in comparison with that in oxygen-free water [3].

Additional information on the ${ }^{1} \mathrm{O}_{2}$ photosensitization efficiency was obtained by analyzing PL transients at $1.63 \mathrm{eV}$ of $\mathrm{Si}-\mathrm{NCs}$ excited by laser pulses. The relaxation time of the exponential part of the PL transients in the solution without $\mathrm{O}_{2}$ was $\tau_{\mathrm{ex}} \approx 85 \mu \mathrm{s}$, which is close to the natural radiative lifetime of excitons confined in $\mathrm{Si}$ NCs. The saturation of the solution with gaseous $\mathrm{O}_{2}$ at a pressure of 760 Torr leads to a decrease in the $\mathrm{PL}$ dacay time to $\tau_{\mathrm{D}} \approx 50 \mu \mathrm{s}$. The ratio $\tau_{\mathrm{ex}} / \tau_{\mathrm{D}} \approx 1.6$ is close to the measured PL quenching. These observations indicate that the decrease of the PL lifetime of Si-NCs in oxygen-saturated suspensions was mainly caused by the photosensitized generation of ${ }^{1} \mathrm{O}_{2}$.

The biomedical experiments in vitro were carried out by using cancer cells of 3T3 NIH (mouse fibroblast) grown by standard procedure of their sub cultivation in the Dulbecco-modified Eagle's medium using 96-well plates or dishes. The cells were cultivated for 1.5 days, and then the medium was changed on the fresh nutrient solution with different concentrations of Si-NCs. One part of the wells was illuminated by mercury lamp, another part was kept in darkness and some cells were used for control (without Si-NCs and illumination). The inhibitory or lethal effect of the illumination was obtained by measuring the cell quantity in nonilluminated and illuminated wells per cells quantity in the control wells in which the Si-NC suspension was not added. Dependence of the cancer cell number (relative to the control) as a function of the concentration of por-Si powder in the solution is given in Figure 8 . The number of survived cells after the irradiation in the growth medium with SiNCs concentration about $0.5 \mathrm{~g} / 1$ decreases strongly as compared to the reference group. The death of $80 \%$ of cells was detected for the concentration higher $2 \mathrm{~g} / \mathrm{l}$. At the same time, the effect of por-Si was almost absent over the entire concentration range in darkness. This fact is concerned with the ${ }^{1} \mathrm{O}_{2}$ generation process under the illumination in the active solution with dispersed $\mathrm{Si}-\mathrm{NCs}$, because ${ }^{1} \mathrm{O}_{2}$ being strong oxidizing substance results in both killing of cells and the inhibitions of cell proliferation. 


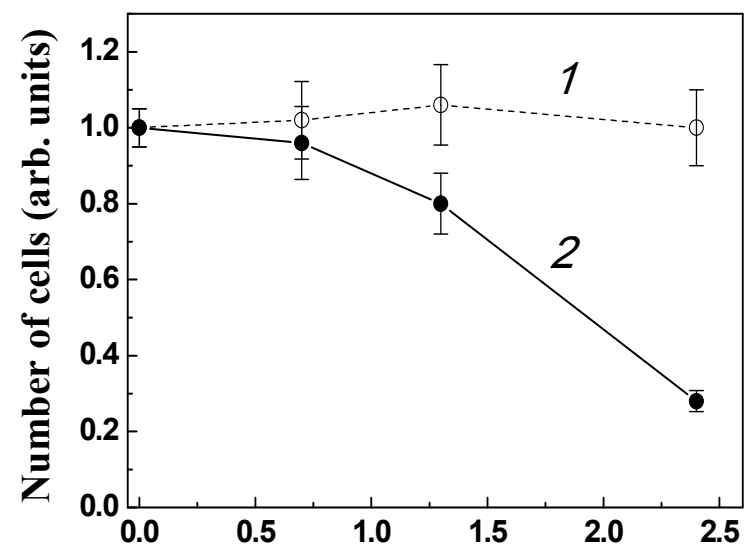

Concentration of nc-Si (g/l)

Figure 8 - Number of cancer cells in darkness (1) and after illumination (2) $v s \mathrm{Si}-\mathrm{NCs}$ concentration in nutrient solution (Ref. [3])

The detected death of cancer cells occurred due likely to the action of photosensitized active oxygen, in particular, to the oxidation of cell substance by ${ }^{1} \mathrm{O}_{2}$. In addition, the effect of other active forms of oxygen as superoxide $\left(\mathrm{O}_{2}{ }^{-}\right.$ions) was also possible [3]. To reveal the particular mechanisms of the interaction between photoexcited Si-NCs and biological objects, additional investigations are still required.

\section{Sensitization of therapeutic ultrasound}

Sonodynamic therapy (SDT) is a method of mild cancer therapy, which is realized by using ultrasonic irradiation (USI) with therapeutic intensity in the range of $1-10 \mathrm{~W} / \mathrm{cm}^{2}$ [8]. SDT usually requires more simple and cheap set-up in comparison with other methods based on high-intensity focused ultrasound [41]. NPs of por-Si were successfully used as sonosensitizers under USI at $37 \mathrm{kHz}$ for the destruction of cancer cells in vitro $[42,43]$. It was found that the NPs activated by USI with relatively low intensity about $0.2 \mathrm{~W} / \mathrm{cm}^{2}$ did not destroy directly the cells, while the cell death occurred for 1-2 days due to the apoptosis. In order to explain the observed effect, the possible mechanisms of the cell death were proposed: (1) local heating (hyperthermia); (2) "nano-scalpel" effect of NPs, which destroy mechanically the cancer cells; (3) sensitization of cavitation, which results in the shock wave generation and additional dissipation of the ultrasound energy [43]. Note, that the USI with frequency in the $\mathrm{kHz}$ range is usually accompanied by strong cavitation and the third mechanism should be dominant. However, the therapeutic USI is commonly operated at the $\mathrm{MHz}$ frequency and all the mechanisms can be important for the sonosensitizing properties of por-Si NPs.

Numerical simulations and physical experiments showed that por-Si NPs with sizes above $100 \mathrm{~nm}$ can efficiently sensitize the hyperthermia under therapeutic USI with frequencies of $1-2.5 \mathrm{MHz}$ and intensities of $1-20 \mathrm{~W} / \mathrm{cm}^{2}$ [9]. It was noted that the dry-ground por-Si NPs exhibited 2 times larger heating than the wet-ground ones and the effect was explained by the large sizes of the former, as well as by hydrophobic properties of their surfaces and, as a consequence, greater viscous friction [9].

USI with frequency at $0.88-2.64 \mathrm{MHz}$ and porSi NPs with sizes about $100 \mathrm{~nm}$ were used to destroy cancer cells in vitro and in vivo. Photoluminescent por-Si NPs, which were composed by smaller Si-NCs covered with biopolymer (dextrane), were efficiently up-taken by cancer cells without any undesired cytotoxic effect in vitro. The uptake of NPs was monitored by using the PL imaging technique [8]. Furthermore, the NP suspensions with doses up to $30 \mathrm{mg} / \mathrm{kg}$ could be easily intravenously injected in vivo. It was found that the combined impact of NPs and USI with intensity of $1-2 \mathrm{~W} / \mathrm{cm}^{2}$ resulted in a strong suppression of the cancer tumor growth as shown in Figure 9 [8]. The observed SDT effect is explained by the both cavitation-induced destruction of cancer cells and local hyperthermia caused by the therapeutic USI and sensitized by Si NPs. 
(a)

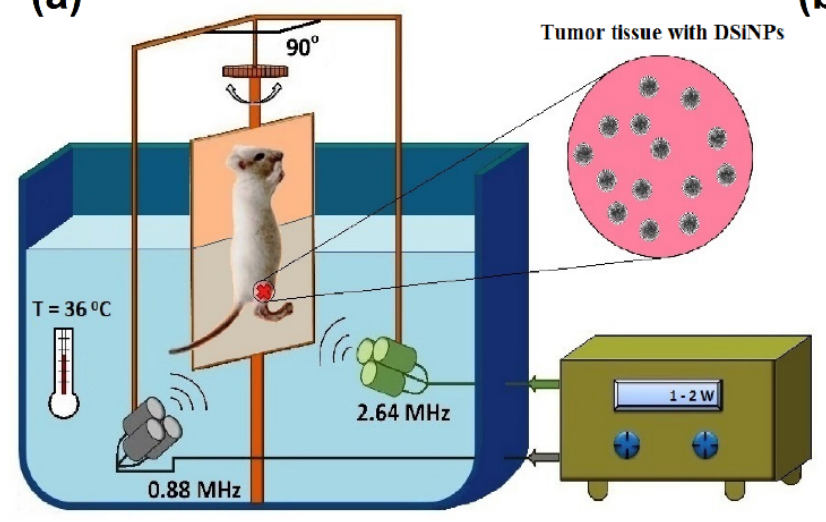

(b)

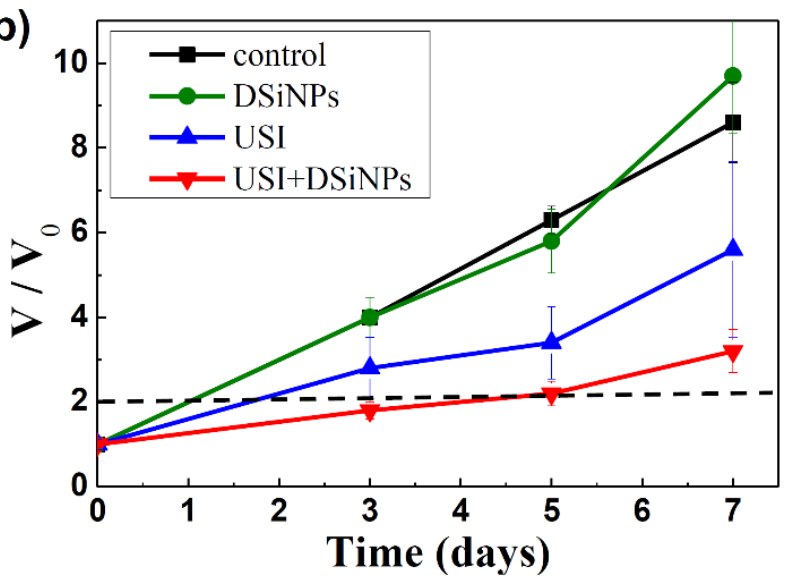

Figure 9 - (a) Schematic image of the experimental setup for USI experiments in vivo.

(b) Dynamics of the cancer tumor growth for mice with intravenously injected dextrane-coated por-Si NPs followed by USI treatment (red down triangles), for mice with intravenously injected por-Si NPs only (green circles), for mice with the USI treatment only (blue up triangles), and for the control group of mice (black squares).

The horizontal dashed line indicates the doubled tumor volume. (Ref. [8])

\section{$5 \quad$ RF-induced hyperthermia}

Recently, Si-NCs and NPs were found to act as efficient sensitizers of electromagnetic radiofrequency $(\mathrm{RF})$ radiation-induced hyperthermia [10]. Due to a large penetration depth of the RF radiation the effect seems to be very promising to treat malignant tumors and even individual cells in metastases. The principle of RF-induced hyperthermia sensitized by $\mathrm{Si}$ NPs is shown schematically in Figure 10a.

The physical experiments showed that heating of aqueous suspensions of PSi NPs by tens of Celsius degrees could be achieved under RF irradiation at $27 \mathrm{MHz}$ with relatively low intensity below $5 \mathrm{~W} / \mathrm{cm}^{2}$ (see Figure 10b). The heating effect was demonstrated for por-Si NPs prepared by mechanical grinding of por-Si layers [8] as well as for non-porous Si NPs synthesized by laser ablation of $\mathrm{c}-\mathrm{Si}$ in water [16]. The observed RF heating effect can be explained by considering the polarization of $\mathrm{Si}$ NPs and electrolyte in the electrical field of RF radiation and the corresponding release of the Joule heat. The polarization of electrolyte ensures relatively high ionic conductivity due to the electric current outside $\mathrm{NP}, I_{\text {out }}$ (see Figure 10a). The higher heating rate for laser-ablated NPs is related by their smaller sizes. The size-dependent specific heating rate per a mass unit of NPs can be estimated by the following expression:

$$
S \sim E^{2} / D^{2},
$$

where $E$ is the amplitude of the RF electrical field and D is the size of NP [10]. It was theoretically shown that the RF heating by the electrical current inside small NP, $I_{\text {in, is }}$ weaker in comparison with $I_{\text {out }}$ because of the significantly larger volume for the heat generation by the latter [44].

In-vivo experiments with mice under therapeutic $\mathrm{RF}$ irradiation were carried out (see Figure 11). The RF setup consisted of a medically approved source at $27 \mathrm{MHz}$ (Figure 11a). Histology images of tumor areas, which were obtained $1 \mathrm{~h}$ and 3 days after the PSi NP injection and RF-based treatment, respectively, indicate both the destruction of cancer cells (visible as dark blue spots) and biodegradation of por-Si NPs in the cells (indicated by red arrows in Figure $11 \mathrm{~b}-\mathrm{c})$. It was found that NPs under activation with RF radiation resulted in $35-40 \%$ suppression of the tumor growth, while NPs themselves can slightly inhibit the tumor growth (Figure 11d). This effect is explained by the toxic effect of free radicals (dangling bonds) from the surface of Si NPs during the dissolution process. Similar slight inhibition of the tumor growth took place under the action of RF radiation alone. However, the combined action of PSi NPs and RF excitation could drastically amplify the effect leading to a much stronger inhibition of the tumor growth. 


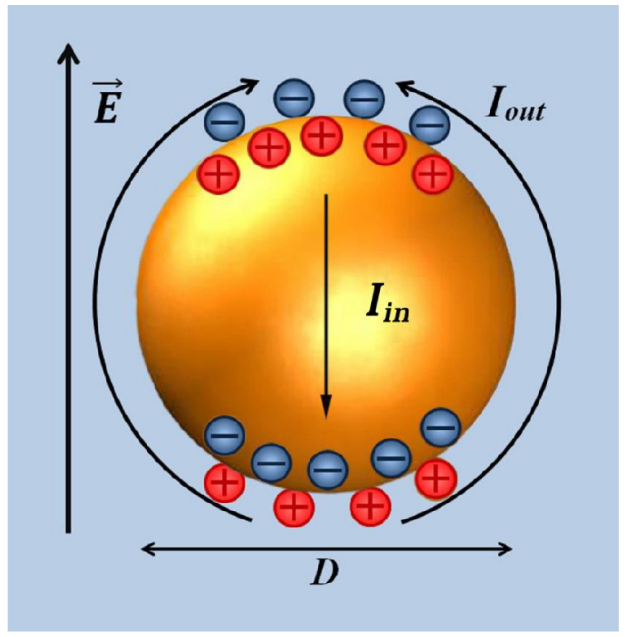

(a)

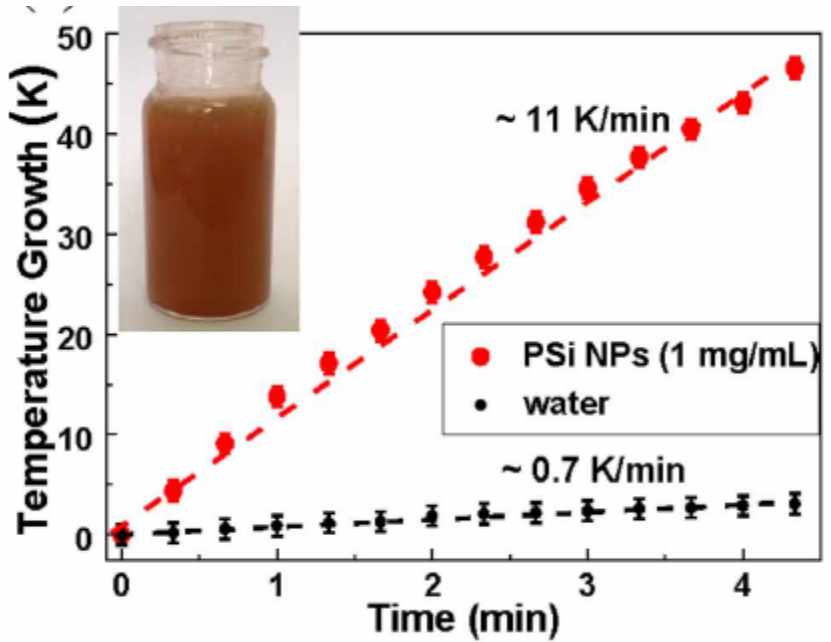

(b)

Figure 10 - (a) Schematic image of the RF-radiation induced electrical currents inside and outside a Si NP and transients of the temperature growth under RF-irradiation of aqueous suspension of por-Si NPs (red circles) and pure water (black circles); inset shows the NP suspension (Ref. [10])

(a)
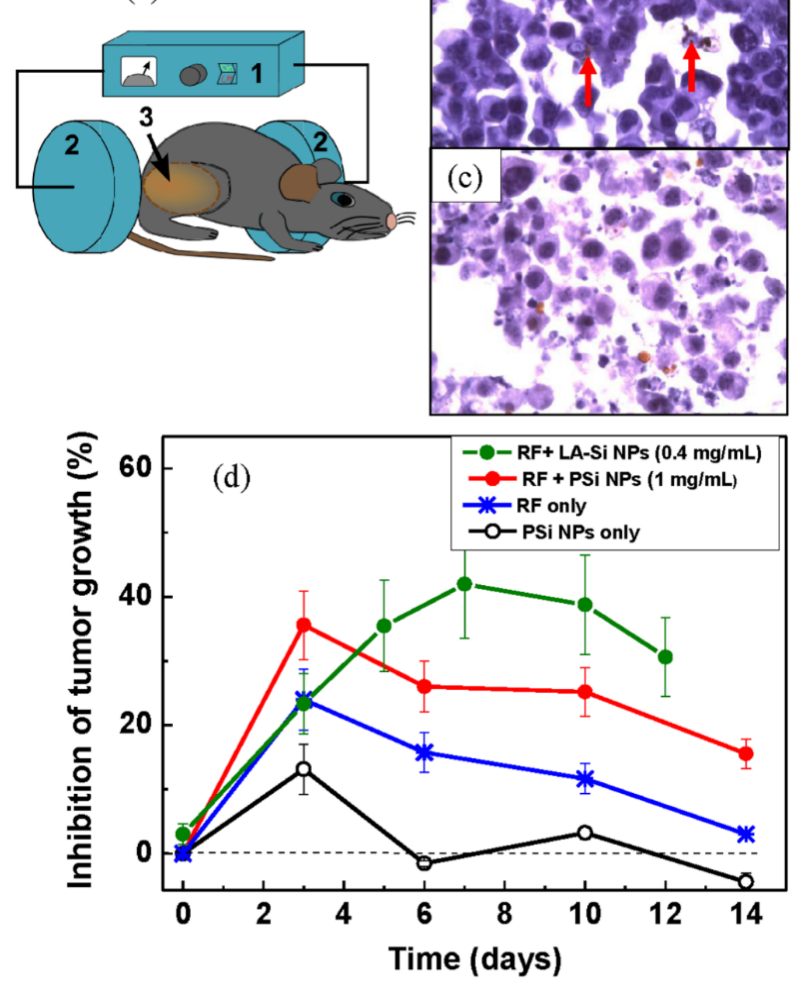

Figure 11 - (a) Schematics of the RF radiation-based therapy setup where (1)

is a RF radiation source, (2) are the RF electrodes, (3) is a mouse having a tumor area.

(b), (c) Histology images of a tumor area $1 \mathrm{~h}$ and 3 days after the PSi NP injection

and RF-based treatment, respectively. (d) Inhibition of the tumor growth after the following treatments: injection of PSi NPs suspension without RF irradiation (black curve);

RF irradiation 2 min without PSi NPs (blue curve); injection of PSi NPs followed by 2 min RF irradiation (red curve); injection of a suspension of laser-ablated (LA-Si) NPs followed by 2 min RF irradiation (green curve). (Ref. [10]) 


\section{Conclusions}

The obtained results and their analysis indicate that Si nanocrystals and nanoparticles are promising sensitizers of the cancer therapy based on the photo. sono- and radiofrequency irradiations. The photosensitization of singlet oxygen generation occurs because of the energy transfer from photoexcited Si nanocrystals to oxygen molecules adsorbed on the nanocrystal surfaces. The singlet oxygen generation was observed for por-Si in different gaseous and liquid ambiences. The effect of photoexcited Si nanocrystals on the cancer cell proliferation was confirmed in vitro. While these observations open a way for in vivo experiments and then to possible applications of por-Si in PDT of malignant tumors, the real progress in this direction is still insignificant. The investigation of physical processes and biomedical experiment in vitro and in vivo show the good prospect of biocompatible and biodegradable Si nanoparticles for the application in sonodynamic therapy of cancer. The RF hyperthermia sensitized by $\mathrm{Si}$ nanoparticles represents the mild method of cancer therapy. Moreover, the sensitizing properties of PSi NPs can be combined with cancer diagnostics by means of the optical bioimaging. These remarkable properties of $\mathrm{Si}$ nanoparticles are very important for their practical implementation in cancer theranostics.

\section{Acknowledgements}

The author is grateful to A.V. Kabashin, L.A. Osminkina, A.A. Kudryavtsev, Yu.V. Ryabchikov, M.B. Gongalsky, and I.A. Belogorokhov for their assistance in the experimental studies presented in this paper. The work was partially supported by the Russian Science Foundation (Grant No16-1310145).

\section{References}

1. L. Pavesi, R. Turan. Silicon nanocrystals: fundamentals, synthesis and applications. Wiley-VCH Verlag GmbH \& Co. KGaA. $-2010 .-622$ p.

2. A.A. Ischenko, G.V. Fetisov, L.A. Aslalnov. Nanosilicon: Properties, Synthesis, Applications, Methods of Analysis and Control. USA: CRC Press, -2014. - 755 p.

3. V.Yu. Timoshenko, A.A. Kudryavtsev, L.A. Osminkina, A.S. Vorontzov, Yu.V. Ryabchikov, I. A. Belogorokhov, D. Kovalev, P.K. Kashkarov. Silicon nanocrystals as photosensitizers of active oxygen for biomedical applications // JETP Lett., - 2006. - Vol. 83. -P. 423-426.

4. N. O'Farrell, A. Houlton, B.R. Horrocks. Silicon particles: applications in cell biology and medicine // Int. J. Nanomed., - 2006. -Vol. 1(4). -P. 451-472.

5. C.M. Gonzalez, M. Iqbal, M. Dasog, D.G. Piercey, R. Lockwood, T.M. Klapötke, J.G.C. Veinot. Detection of high-energy compounds using photoluminescent silicon nanocrystal paper based sensors // Nanoscale, - 2014. -Vol. 6. -P. 2608-2612.

6. J.-H. Park, L. Gu, G. v. Maltzahn, E. Ruoslahti, S.N. Bhatia, M.J. Sailor. Biodegradable luminescent porous silicon nanoparticles for in vivo applications // Nat. Mater., - 2009. -Vol. 8. -P. 331-336.

7. R. Jugdaohsingh. J. Nutr. Silicon and bone health // J. Natr. Health Aging. - 2007. - Vol. 11(2). -P. 99-110.

8. L.A. Osminkina, A.L. Nikolaev, A.P. Sviridov, N.V. Andronova, K.P. Tamarov, M.B. Gongalsky, A.A. Kudryavtsev, H.M. Treshalina, V.Yu. Timoshenko. Porous silicon nanoparticles as efficient sensitizers for sonodynamic therapy of cancer // Micropor. \& Mesopor. Mater., -2015. - Vol. 210. -P. 169-175.

9. A.P. Sviridov, V.G. Andreev, E.M. Ivanova, L.A. Osminkina, K.P. Tamarov, V.Yu. Timoshenko. Porous silicon nanoparticles as sensitizers for ultrasonic hyperthermia // Appl. Phys. Lett., - 2013. -Vol. 103. - P. 193110.

10. K.P. Tamarov, L.A. Osminkina, S.V. Zinovyev, K.A. Maximova, J.V. Kargina, M.B. Gongalsky, Yu. Ryabchikov, A. Al-Kattan, A.P. Sviridov, M. Sentis, A.V. Ivanov, V.N. Nikiforov, A.V. Kabashin, V.Yu. Timoshenko. Radio frequency radiation-induced hyperthermia using Si nanoparticle-based sensitizers for mild cancer therapy // Sci. Rep., -2014. - Vol.4. - P. 7034.

11. M.B. Gongalsky, A.Yu. Kharin, S.A. Zagorodskikh, L.A. Osminkina, V.Yu. Timoshenko. Photosensitized generation of singlet oxygen in porous silicon studied by simultaneous measurements of luminescence of nanocrystals and oxygen molecules // J. Appl. Phys. -2011. -Vol. 110. - P. 013707.

12. E.J. Anglin, L. Cheng, W.R. Freeman, M.J. Sailor. Porous silicon in drug delivery devices and materials // Adv. Drug. Deliv. Rev., -2008. -Vol. 60. -P. 1266-1277.

13. H.A. Santos, J. Hirvonen. Nanostructured porous silicon materials: potential candidates for improving drug delivery // Nanomedicine, - 2012. -Vol. 7. -P. 1281-1284. 
14. A. Yurtsever, M. Weyland, D.A. Muller. Three-dimensional imaging of nonspherical silicon nanoparticles embedded in silicon oxide by plasmon tomography // Appl. Phys. Lett. -2006. - Vol. 89. -P. 151920.

15. L. Gu, D.J. Hall, Zh. Qin, E. Anglin, J. Joo, D.J. Mooney, S.B. Howell, M.J. Sailor. In vivo time-gated fluorescence imaging with biodegradable luminescent porous silicon nanoparticles // Nat. Commun., - 2013. -Vol. 4. P. 2326.

16. A. V. Kabashin, V. Yu. Timoshenko. What theranostic applications could ultrapure laser-synthesized Si nanoparticles have in cancer? // Nanomedicine, -2016. -Vol. 11(17). -P. 2247-2250.

17. M. B. Gongalsky, L.A. Osminkina, A. Pereira, A. A. Manankov, A. A. Fedorenko, A.N. Vasiliev, V. V. Solovyev, A. A. Kudryavtsev, M. Sentis, A. V. Kabashin, V. Yu. Timoshenko. Laser-synthesized oxide-passivated bright Si quantum dots for bioimaging // Sci. Rep., -2016. -Vol. 6. - P. 24732.

18. A. Uhlir. Electrolytic shaping of germanium and silicon // Bell Syst. Tech., -1956. -Vol. 35. -P. 333.

19. A.G. Cullis, L.T. Canham, P.D.J. Calcott. Desorption-ionization mass spectrometry on porous silicon // J. Appl. Phys., -1997. -Vol. 82. -P. 965.

20. O. Bisi, S. Ossicini, L. Pavesi. Porous silicon: a quantum sponge structure for silicon based optoelectronics // Surf. Sci. Rep., -2000. -Vol. 38. -P. 1-126.

21. D. Kovalev, H. Heckler, G. Polisski, F. Koch. Scaleable synthesis route for silicon nanocrystal assemblies // Phys. Stat. Sol. (b), - 1999. - Vol. 215. -P. 871.

22. G.D. Sanders, Y.-C. Chang. Theory of optical properties of quantum wires in porous silicon // Phys. Rev. B,1992. - Vol. 45.-P. 9202.

23. B. Delley, E.F. Steigmeier. Size dependence of band gaps in silicon nanostructures // Appl. Phys. Lett. -1995. Vol. 67. -P. 2370.

24. G.C. John, V.A. Singh. Porous silicon: theoretical studies // Phys. Rep., - 1995. -Vol. 263. -P. 93-151.

25. D.A.G. Bruggeman. Berechnung verschiedener physikalischer konstanten von heterogenen substanzen. I. Dielektrizitätskonstanten und leitfähigkeiten der mischkörper aus isotropen substanzen // Ann. der Phys. -1935. -Vol. 24. - P. 636

26. A.N. Obraztsov, V. Yu. Timoshenko, H. Okushi, H. Watanabe. Photoacoustic spectroscopy of porous silicon // Semicond., -1997. -Vol. 31. - P. 534.

27. D. G. Yarkin, E.A. Konstantinova, V.Yu. Timoshenko. Investigation of the photovoltage in por-Si/p-Si structures by the pulsed-photovoltage method // Semicond., - 1995. -Vol.29. -P. 348.

28. Yu.V. Ryabchikov, I.A. Belogorokhov, A.S. Vorontsov, L.A. Osminkina, V.Yu. Timoshenko, P.K. Kashkarov. Dependence of the singlet oxygen photosensitization efficiency on morphology of porous silicon // Phys. stat. sol. (a), 2007. -Vol. 204. -P. 1271.

29. E.A. Konstantinova, V.A. Demin, A.S. Vorontzov, Yu.V. Ryabchikov, I.A. Belogorokhov, L.A. Osminkina, P.A. Forsh, P.K. Kashkarov, V.Yu. Timoshenko. Electron paramagnetic resonance and photoluminescence study study of dilicon nanocrystals-phorosensitizers of singlet oxygen molecules //J. Non-Cryst. Sol., -2006. -Vol. 352. -P. 11561159 .

30. E.A. Konstantinova, V.A. Demin, V.Yu. Timoshenko, P.K. Kashkarov. EPR diagnostics of the photosensitized generation of singlet oxygen on the surface of silicon nanocrystals // JETP Lett., - 2007. -Vol. 85. - P. 59.

31. E.A. Konstantinova, V.A. Demin, V.Yu. Timoshenko. Investigation of the generation of singlet oxygen in ensembles of photoexcited silicon nanocrystals by electron paramagnetic resonance spectroscopy // J. Exp. Theor. Phys., -2008. -Vol. 107. - P. 473-481.

32. S.J. Arnold, M.Kubo, E.A. Ogryzlo. Relaxation and reactivity of singlet oxygen // Advan. Chem. Ser., - 1968. Vol. 77. -P. 133-142.

33. J.G. Moser. Photodynamic tumor therapy: 2nd and 3rd Generation Photosensitizers. - Harwood Academic Publishers. - Amsterdam. - 1998. -242 p.

34. N. J Turro, Modern molecular photochemistry. -University Science. -Sausalito, CA. -1991. - 628 p.

35. D.R. Kearns. Physical and chemical properties of singlet molecular oxygen // Chem. Rev. -1971. -Vol. 71. -P. 395-427.

36. D. Kovalev, E. Gross, N. Künzner, F. Koch, V.Yu. Timoshenko, M. Fujii. Resonant electronic energy transfer from excitons confined in silicon nanocrystals to oxygen molecules // Phys. Rev. Lett., - 2002. -Vol. 89. -P. 137401.

37. E. Gross, D. Kovalev, N. Künzner, F. Koch, V. Yu. Timoshenko, M. Fujii. Spectrally resolved electronic energy transfer from silicon nanocrystals to molecular oxygen mediated by direct electron exchange // Phys. Rev. B, 2003. -Vol. 68. -P. 115405.

38. M. Fujii, M. Usui, Sh. Hayashi, E. Gross, D. Kovalev, N. Künzner, J. Diener, V.Yu. Timoshenko. Chemical reaction mediated by excited states of Si nanocrystals-Singlet oxygen formation in solution // J. Appl. Phys., -2004. Vol. 95. - P. 3689.

39. D. Kovalev, M. Fujii. Silicon nanocrystals: photosensitizers for oxygen molecules // Adv. Mat. - 2005. -Vol. 17. -P. 2531.

40. V. Chirvony, V. Bolotin, E. Matveeva, V. Parkhutik. Fluorescence and $1 \mathrm{O} 2$ generation properties of porphyrin 
molecules immobilized in oxidized nano-porous silicon matrix // J. Photochem. \& Photobilogy: Chem., -2006. -Vol. 181. -P. 106.

41. N. Yumita, N. Okuyama, K. Sasaki, S. Umemura. Sonodynamic therapy on chemically induced mammary tumor: pharmacokinetics, tissue distribution and sonodynamically induced antitumor effect of gallium-porphyrin complex ATX-70 // Cancer Chemother. Pharmacol., - 2007. -Vol. 60. - P. 891 - 897.

42. L.A. Osminkina, M.B. Gongalsky, A.V. Motuzuk, V.Yu. Timoshenko, A.A. Kudryavtsev. Silicon nanocrystals as photo- and sono-sensitizers for biomedical applications //Appl. Phys. B: Lasers \& Optics, - 2011. -Vol. 105 (3). -P. 665.

43. L.A. Osminkina, E.N. Luckyanova, M.B. Gongalsky, A.A. Kudryavtsev, A.Kh. Gaydarova, R.A. Poltavtseva, P.K. Kashkarov, V.Yu. Timoshenko, G.T. Sukhikh. Effects of nanostructurized silicon on proliferation of stem and cancer cell // Bull. Exper. Biol. \& Med.: Nanotechnol., -2011. -Vol. 151. - P.79.

44. K. P. Tamarov, A. P. Kanavin, V.Yu. Timoshenko, A.V. Kabashin, I.N. Zavestovskaya. Modeling of heat release in aqueous suspensions of solid-state nanoparticles under electromagnetic radio-frequency irradiation // Proc. SPIE, -2016. -Vol. 9737. - P.973706. 